\title{
IEEJ Industry Applications Society
}

\section{《BOARD OF SOCIETY 》}

PRESIDENT

EDITOR IN CHIEF (JAPANESE JOURNAL)

EDITOR IN CHIEF (ENGLISH JOURNAL)

VICE PRESIDENTS

OFFICERS, PlanNING \& GENERAL AFFAIRS

OFFICERS, TREASURERS

OFFICERS, EDITORIAL AFFAIRS

OFFICERS, R\&D MANAGEMENT

OFFICERS, INTERNATIONAL AFFAIRS

\author{
KAZUNOBU OHYAMA (Daikin Industries) \\ TOSHIYUKI MURAKAMI (Keio Univ.) \\ KIYOSHI OHISHI (Nagaoka Univ. of Tech.) \\ KAZUMASA IDE (Hitachi Power Solutions) \\ MASAYUKI MORIMOTO (Tokai Univ.) \\ SHOJI SHIMOMURA (Shibaura Inst. of Tech.) \\ MasaAKI Shibata (Seikei Univ.) \\ MASAKI EGUCHI (Sharp) \\ YASUShi MATSUMOTO (Fuji Electric) \\ KEIICHIRO KONDO (Chiba Univ.) \\ HARUYUKI KOMETANI (Mitsubishi Electric) \\ NORIKO KAWAKAMI (TMEIC) \\ TAKASHI KOSAKA (Nagoya Inst. of Tech.) \\ SHINZO TAMAI (TMEIC) \\ TOMOKI YOKOYAMA (Tokyo Denki Univ.)
}

AUDITORS HIDEAKI FUJITA (Tokyo Inst. of Tech.)

OFFICERS KAN AKATSU (Shibaura Inst. of Tech.)

ATSUO KAWAMURA (Yokohama National Univ.)

YUKIHIKO SATO (Chiba Univ.)

KENTARO SUZUKI (Toshiba)

RYOJI MizUTANI (Toyota Motor)

\section{《R\&D STEERING COMMITTEE 》}

CHAIRPERSON

VICE CHAIRPERSONS

$\underline{\text { SECRETARIES }}$

MEMBERS

KAZUMASA IDE (Hitachi Power Solutions)

NORIKO KAWAKAMI (TMEIC)

SeiICHIRO Katsura (Keio Univ.)

Hiroshi Igarashi (Tokyo Denki Univ.)

Motohiro KAWAFUKU (Daido Univ.)

Atsuhiko Kuzumaki (Toshiba)

Tatsuo Teratani (Nagoya Univ.)

KATSUHIKO INAGAKI (Tokai Univ.)

TOSHIHIKo ODA (VICS Center)

Junichi Kitano (Central Japan Railway)

NobUhisa KobaYashi (Hitachi)

Masaaki Shibata (Seikei Univ.)

TADASHI FUKAMI (Kanazawa Inst. of Tech.)

Hideaki Minakata (Chiba Inst. of Tech.)

TOMOKI WATANABE (Tokyo Inst. of Tech.)

\section{《Global ACtivity CommitTeE 》}

CHAIRPERSON

VICE CHAIRPERSON

SECRETARIES

MEMBERS
Shinzo TAMAI (TMEIC)

Tомокі YoкоYама (Tokyo Denki Univ.)

KAN AKATSU (Shibaura Inst. of Tech.)

KIYOSHI OHISHI (Nagaoka Univ. of Tech.)

MASAKI EgUCHI (Sharp)

SeitChiro Katsura (Keio Univ.)

Fujio Kurokawa (Nagasaki Univ.)

Nobuo SATOH (Chiba Inst. of Tech.)

Toshinisa SHIMIZu (Tokyo Metropolitan Univ.)

TsUYOSHI HiguCHI (Nagasaki Univ.)

Mikiniko Matsui (Tokyo Polytechnic Univ.)

TAKAShi Yamashita (NTT Facilities Research Inst.)
YOSUKE NAKAZAWA (Toshiba)

KOZO IDE (Yaskawa Electric)

TAKAFUMI KOSEKI (The Univ. of Tokyo)

TOSHIHISA SHIMIZU (Tokyo Metropolitan Univ.)

TADATOSHI BABASAKI (NTT Facilities)

HideAKI MinaKaTA (Chiba Inst. of Tech.)

TAKASHI KosAKA (Nagoya Inst. of Tech.)

YuJI ENOMOTO (Hitachi)

TAKAYUKI KaShiWAGI (RTRI)

MASAHIRo Kimata (Mitsubishi Electric)

Ryogo Kubo (Keio Univ.)

Hitoshi Haga (Nagaoka Univ. of Tech.)

HIDEKI OMORI (Osaka Inst. of Tech.)

TAKEO KANAI (TMEIC)

Hisao Kubota (Meiji Univ.)

RYOICHI SHIOBARA (Hitachi)

AKIRA Shimada (Shibaura Inst. of Tech.)

RyOJi Mizutani (Toyota Motor)

TAKAYUKI MURAyaMa (Tokyo Waterworks Bureau)
KENJI NATORI (Chiba Univ.)

Toshiyuki Murakami (Keio Univ.)

HIROYUKI OHSAKI (The Univ. of Tokyo)

Hisao Kubota (Meiji Univ.)

SuzuO SAITo (Toshiba)

MaSAAKI Shibata (Seikei Univ.)

YASUYUKI NishIDA (Chiba Inst. of Tech.)

YOICHI HORI (The Univ. of Tokyo)

ICHIRO MIKI (Meiji Univ.) 


\section{《 Editorial Committee 》}

CHAIRPERSON MASAYUKI MORIMOTO (Tokai Univ.)

Vice ChaIrPERSONS KeIICHIRO Kondo (Chiba Univ.)

SECRETARIES $\quad$ RYogo KUBo (Keio Univ.)

MEMBERS Masafumi MiYatake (Sophia Univ.)

YuJI ENOMOTO (Hitachi)

ShUn TANiguChI (Toshiba)

Daisuke Yashiro (Mie Univ.)

NAOKI MOTOI (Kobe Univ.)

YUKINORI INOUE (Osaka Prefecture Univ.)

YUKI HASEGAWA (Saitama Univ.)

Masataka AKagi (RTRI)

MasahIKo TSUKaKoshi (TMEIC)

TAKAHIRO SUZUKI (Hitachi)

YOHEI TORIUMI (NTT)

HIDEAKI ENOKI (Hitachi)

YUKI YOKOKURA (Nagaoka Univ. of Tech.)

KeNJi NATORI (Chiba Univ.)

KAZUKI FUJimoto (Toyo Denki Seizo)

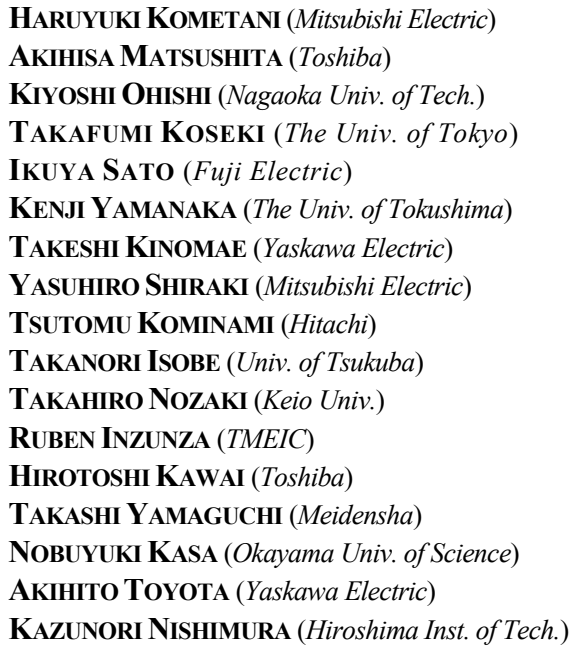

\title{
《IEEJ Transactions on Industry Applications (In JAPAnese) Program Committee 》
}

\author{
Editor In Chief $\quad$ Toshiyuki Murakami (Keio Univ.) $\quad$ VICE Chief $\quad$ Satoshi Suzuki (Tokyo Denki Univ.)
}

《SEMICONDUCTOR POWER CONVERTER (D1) 》

CHAIRPERSON KAZUAKI Mino (Murata Manufacturing)

SECRETARIES $\quad$ JUN-ICHI ITOH (Nagaoka Univ. of Tech.)

Vice Chairperson Toshimitsu Morizane (Osaka Inst. of Tech.)

JUN-ICHI ITOH (Nagaoka Univ. of Tech.) NOBUKAZU HoshI (Tokyo Univ. of Science)

SARI MAEKAwa (Toshiba)

KEIJI WADA (Tokyo Metropolitan Univ.)

《INDUSTRIAL InSTRUMENTATION AND CONTROL, MEChatronics CONTROL (D2)》

Chairperson $\quad$ Satoshi Komada (Mie Univ.) $\quad$ Vice Chairperson Yasue Mitsukura (Keio Univ.)

$\underline{\underline{\text { SeCretaries }}} \quad$ Takenori Atsumi (HGST Japan)

YUTAKA UCHIMURA (Shibaura Inst. of Tech.)

Hiroshi Fujimoto (The Univ. of Tokyo)

《 Rotating Machinery, Motor Drive, Linear Drives (D3) 》

CHAIRPERSON ISAO HiROTSUKA (Chubu Univ.)

SECRETARIES KAN AKATSU (Shibaura Inst. of Tech.)

YASUAKI SAKAMOTO $(R T R I)$

SHU YAMAMOTO (Polytechnic Univ.)

Vice ChairPerson Mimpei Morishita (Kogakuin Univ.)

YUJI ENOMOTO (Hitachi)

KICHIRO YAMAMOTO (Kagoshima Univ.)

YASUHIRO YAMAMOTO (Meidensha)

《Vehicle Technology, Intelligent Transport Systems, Home and Consumer Appliances (D4) 》

CHAIRPERSON

KAZUAKI YUKI (Toshiba)

Vice Chairperson Masafumi Miyatake (Sophia Univ.)

SeCretaries Masayuki Sanada (Osaka Prefecture Univ.)

SATOSHI TAKAHASHI (Nagoya Electric Works)

« Monozukuri, Innovative Industrial System, Transportation and Electric Railway, Smart Facilities, Public Plant ENGINEERING (D5) 》

CHAIRPERSON

SECRETARIES
KAZUAKI YUKI (Toshiba)

NORIMITSU ICHIKAWA (Kogakuin Univ.)

TAKAFUMI KOSEKI (The Univ. of Tokyo)

OSAMU YAMANAKA (Toshiba)
Vice Chairperson Masafumi Miyatake (Sophia Univ.)

Teruo UsaMI (Kyoto Gakuen Univ.)

KENJI SUZUKI (Univ. of Tsukuba)

\section{《 IEEJ JOURNAL OF INDUSTRY APPLICATIONS EDITORIAL BOARD 》}

EDITOR IN CHIEF KIYoshi OHISHI (Nagaoka Univ. of Tech.)

《 Power Electronics And Its Applications (D6) 》

Co-EDITOR IN CHIEF TOMOKI Yokoyama (Tokyo Denki Univ.)

AsSOCIATE EDITOR Hitoshi HAGA (Nagaoka Univ. of Tech.)

AsSOciate EdITOR Hideaki FuJITA (Tokyo Inst. of Tech.)

《Motion CONTRol, Robotics, SENSING AND THEIR APPliCATIONS (D7) 》

Co-Editor In Chief Masaaki Shibata (Seikei Univ.)

ASSOCIATE EDITOR JUN ISHIKAWA (Tokyo Denki Univ.)

Associate Editor Yasutaka Fujimoto (Yokohama National Univ.)

Hiroshi Fujimoto (The Univ. of Tokyo)

TADANAO ZANMA (Chiba Univ.)

《 EleCtric MaChine, Motor Drive AND THEIR APPliCATIONS (D8) 》

Co-Editor In Chief Hisao Kubota (Meiji Univ.)

Associate EdITOR KeIICHIRO Kondo (Chiba Univ.)

Associate Editor TAKashi KosaKa (Nagoya Inst. of Tech.)

AкIO Tова (Fuji Electric)

Masayuki SANAda (Osaka Prefecture Univ.) 